\title{
Diacronie
}

Studi di Storia Contemporanea

$N^{\circ} 34,2$ | 2018

Scuola e società in Italia e Spagna tra Ottocento e Novecento

\section{Johann Chapoutot, Il nazismo e l'Antichità}

\section{Alberto Scigliano}

\section{(2) OpenEdition}

Journals

Edizione digitale

URL: http://journals.openedition.org/diacronie/7950

DOI: $10.4000 /$ diacronie. 7950

ISSN: 2038-0925

Editore

Association culturelle Diacronie

Notizia bibliografica digitale

Alberto Scigliano, « Johann Chapoutot, I/ nazismo e I'Antichità », Diacronie [Online], № 34, 2 | 2018 ,

documento 15, Messo online il 29 juin 2018, consultato il 23 septembre 2020. URL : http://

journals.openedition.org/diacronie/7950; DOI : https://doi.org/10.4000/diacronie.7950 


\title{
Diacronie
}

Studi di Storia Contemporanea

34, $2 / 2018$

Scuola e società in Italia e Spagna tra Ottocento e Novecento

\section{RECENSIONE: Johann CHAPOUTOT, Il nazismo e l'Antichità, Torino, Einaudi, 2017, 523 pp.}

\author{
A cura di Alberto SCIGLIANO
}

Per citare questo articolo:

SCIGLIANO, Alberto, «Johann CHAPOUTOT, Il nazismo e l'Antichità, Torino, Einaudi, 2017, 523 pp.», Diacronie. Studi di Storia Contemporanea : Scuola e società in Italia e Spagna tra Ottocento e Novecento, 34, 2/2018, 29/06/2018,

URL: < http://www.studistorici.com/2018/06/29/scigliano_numero_34/ >

Diacronie Studi di Storia Contemporanea $\rightarrow$ http://www.diacronie.it

Rivista storica online. Uscita trimestrale.

redazione.diacronie@hotmail.it

Comitato di direzione: Naor Ben-Yehoyada - João Fábio Bertonha - Christopher Denis-Delacour - Maximiliano Fuentes Codera Anders Granås Kjøstvedt - John Paul Newman - Deborah Paci - Niccolò Pianciola - Spyridon Ploumidis - Wilko Graf Von Hardenberg

Comitato di redazione: Jacopo Bassi - Luca Bufarale - Gianluca Canè - Luca G. Manenti - Fausto Pietrancosta - Alessandro Salvador - Matteo Tomasoni - Luca Zuccolo

Diritti: gli articoli di Diacronie. Studi di Storia Contemporanea sono pubblicati sotto licenza Creative Commons 3.0. Possono essere riprodotti e modificati a patto di indicare eventuali modifiche dei contenuti, di riconoscere la paternità dell'opera e di condividerla allo stesso modo. La citazione di estratti è comunque sempre autorizzata, nei limiti previsti dalla legge. 


\title{
15/ RECENSIONE: Johann CHAPOUTOT, Il nazismo e l'Antichità, Torino, Einaudi, 2017, 523 pp.
}

\author{
A cura di Alberto SCIGLIANO
}

Il giovane e talentuoso storico francese Johann Chapoutot, docente di storia tedesca all'Università di Paris III - Sorbonne nouvelle si è occupato principalmente dell'aspetto culturale del nazismo, dedicando i suoi studi ai processi di trasformazione etica e culturale operati dal regime hitleriano fra gli anni Trenta e Quaranta del secolo scorso ${ }^{1}$. Le ricerche di Chapoutot, all'interno del più ampio contesto degli studi dedicati all'esperienza nazista, sono dotati però di un taglio più peculiare.

Se è vero che gli storici che s'interessano di nazismo negli ultimi tempi hanno preferito, forse anche sensazionalisticamente, mettere in risalto gli aspetti più esoterici e mistici del credo hitleriano, hanno parimenti tralasciato l'indagine - salvo alcune rare eccezioni - verso il metodico interesse che i nazisti nutrivano per la cultura e la riscoperta delle civiltà classiche. In tal senso Il nazismo e l'Antichità di Chapoutot s'inserisce bene nella storiografia dedicata al nazismo perché ne illumina un punto dell'aspetto culturale spesso, e a torto, tenuto in indebita penombra. Soffermandoci su un banale prospetto di percentuali, decine di volumi e articoli hanno messo a fuoco l'interesse per l'occulto e l'ancestralità ariana del popolo germanico espressa da Hitler o da Himmler, ma pochi, di contro, hanno studiato in maniera sistematica quanto la cultura grecoromana abbia giuocato un ruolo centrale nella fabbricazione identitaria della Germania nazista. Escludendo i lavori ormai datati di Philippe Lacoue-Labarth, Le mythe nazi ${ }^{2}$ e Heidegger, Art and Politics; ${ }^{3}$ Hitler and the power of Aesthetics di Frederic Spotts ${ }^{4}$ e il classico articolo di Susan Sontag

\footnotetext{
${ }^{1}$ CHAPOUTOT, Johann, La legge del sangue, Torino, Einaudi, 2016 [ed. orig.: La Loi du sang: penser et agir en nazi, Paris, Gallimard, 2014]; ID., La révolution culturelle nazie, Paris, Gallimard, 2017.

${ }^{2}$ LACOUE-LABARTH, Philippe, Il mito nazi, Genova, Il Melangolo, 1992 [ed. orig.: Le mythe nazi, Paris, Éd. de l'Aube, 1991].

${ }^{3}$ ID., Heidegger, La finzione del politico. Heidegger, l'arte e la politica, Genova, Il Melangolo, 1991 [ed. orig.: Art and Politics: The Fiction of the Political, Oxford, Blackwell, 1990].

${ }^{4}$ SPOTTS, Frederic, Hitler e il potere dell'estetica, Milano, Johan \& Levy, 2012 [ed. orig.: Hitler and the Power of
} 
Fascinating Fascism apparso originariamente nel 1974 sulla «New York Review of Books» ${ }^{5}$, che purtuttavia non analizzavano a pieno il rapporto tra élite nazista e cultura classica, con Chapoutot siamo di fronte a un volume che a conti fatti mancava nel panorama della storiografia di specie.

Già nel 2008 lo storico francese si era soffermato sulla costruzione del mito nazista dell'antico ${ }^{6}$, suggerendo un'originale chiave di lettura del fenomeno come una forma di mitopoiesi tipicamente hitleriana. Nell'articolo veniva illustrato in che modo, all'interno del pensiero del Führer, il Reich non sarebbe stato nient'altro che la riproposizione contemporanea dell'epopea romana. I tedeschi, temprati dal razzismo, non avrebbero però permesso la contaminazione del sangue che, nello schema ideologico nazista, aveva condotto alla rovina l'impero dei Cesari ${ }^{7}$. Questi e altri argomenti sono stati dunque sviluppati in maniera più sistematica e approfondita ne Il nazismo e l'Antichità, in cui il quesito d'indagine principale è quello di capire e scrutare le modalità attraverso le quali i nazisti guardavano non soltanto la civiltà classica mediterranea, ma anche le grandi civiltà dell'antico Oriente. Nel suo libro, suddiviso in nove capitoli raggruppati in tre parti, Chapoutot ricostruisce difatti la concezione nazista di Weltgeschichte intesa però come storia delle razze, di cui quella nordica ricopre il ruolo centrale e più alto, Herrenrasse totale, e del necessario e ineluttabile conflitto fra esse. La gerarchia nazionalsocialista, militare e culturale che fosse, coltivò la precisa intenzione di ricondurre ai popoli del nord la genesi di ogni altra civiltà antica. Attraverso varie pubblicazioni, che Chapoutot presenta meticolosamente, si evince come il paradigma nazista, supportato da numerosi accademici, fosse incentrato sulla ricostruzione mitica di un popolo matrice, quello ario-germanico, che grazie alle migrazioni verso sud fu artefice della nascita della civiltà classica e, addirittura, di quella mesopotamica e indiana. Ma vi si leggono anche le conseguenze reali di queste premesse ideologiche. Dal momento che, alla prova dei fatti, risultava chiaro quanto i caratteri somatici dei popoli del bacino del Mediterraneo e del vicino Oriente fossero lontani da quelli nordeuropei mitizzati nella retorica razzista, l'autore dimostra che gli ideologi nazisti usarono quest'evidenza tanto come prova schiacciante del pericolo della mescolanza razziale, quanto come necessità geopolitica di riportare un dominio tedesco su quelle terre che, secondo tale idea, germaniche lo furono, almeno razzialmente, nell'antichità.

Cionondimeno uno degli snodi centrali e più degni d'attenzione del lavoro dello storico francese è la ricostruzione della contrapposizione fra le due anime del nazismo. Se da una parte Hitler e i cultori della classicità difesero strenuamente il rapporto biunivoco fra Grecia e

Aesthetics, London, Hutchinson, 2002].

${ }^{5}$ SONTAG, Susan, Fascinating Fascism, in EAD., Under the Sign of Saturn, New York, Vintage Books Editions, 1980, pp. 73-107.

${ }^{6}$ CHAPOUTOT, Johann, «Comment meurt un Empire: le nazisme, l'Antiquité et le mythe», in Revue Historique, 647, 2008, pp. 657-676.

${ }^{7}$ Ibidem, p. 665. 
Germania, dall'altra Himmler e l'apparato delle SS si concentrarono invece sulla ricerca dell'eredità dei popoli che erano vissuti sul territorio tedesco, promuovendo attività archeologiche e pubblicazioni dedicate. Ricerche non sempre apprezzate e viste di buon occhio dallo stesso Hitler, dacché parevano dimostrare la profonda differenza rispetto a ciò che l'archeologia riportava alla luce nelle zone latine e greche. Per Hitler i tedeschi avevano un passato subalterno se paragonato alla classicità; la germanicità esasperata di Himmler, che restava estasiato davanti a resti di capanne e a miseri oggetti in terracotta, dimostrava che «quando la Grecia e Roma avevano ormai raggiunto un livello culturale altissimo, noi eravamo bravi a lasciare soltanto asce di pietra e starcene accovacciati intorno ai fuochi» ${ }^{8}$. Per questo motivo Hitler invogliò una vera e propria opera di assimilazione culturale: il passato della razza tedesca, in un rapporto tangente di continuità, era da rintracciare fra il Partenone e il Colosseo. Il rapporto personale di Hitler con l'antichità più che di ammirazione si muoveva secondo coordinate di una vera e propria infatuazione spasmodica e ossessiva. Cosa poteva essere meglio della disciplina militare di Sparta per costruire il nuovo soldato politico nazista pronto a colonizzare il mondo? E non forniva Roma l'esempio migliore per costruire un impero monumentale? Chapoutot ne ricostruisce la retorica attraverso l'analisi della stampa del periodo, anche quella accademica, che si dimostrò a conti fatti del tutto assoggettata ai dettami politici dell'élite nazionalsocialista.

L'antichità classica era dunque assimilata alla germanicità come perpetuazione meridionale della razza nordica e tedeschi, greci e romani appartenevano tutti al medesimo ceppo razziale. $\mathrm{Ne}$ consegue allora che la memoria monumentale del passato classico, eroico e mitico, era stato il florilegio dell'intelletto degli uomini che migrarono da nord. Chapoutot illustra infatti come per Hitler e i classicisti tedeschi il rapporto con il patrimonio culturale dell'antichità fosse decodificato attraverso una successione naturale ad Atene e Roma, in un contesto millenario in cui i nemici da affrontare erano rimasti immutati. Cristianesimo, capitalismo ebraico e bolscevismo erano gli ultimi latori di quegli stessi principii semitici che avevano portato alla rovina la cultura greca e quella latina.

La Francia e l'Italia, che in momenti diversi e a varie intensità avocavano a sé l'eredità latina, erano rispettivamente un nemico e un esempio, laddove però, nel delirio di onnipresenza germanica di Himmler, gli italiani erano visti con sospetto dal momento che, pur conservando nei loro musei esempi dell'arianità dei romani, «non hanno alcun interesse per queste cose» ${ }^{9}$. In tal senso il nazismo sarebbe stato invece il nuovo umanesimo, politico e razziale, che avrebbe reinventato la paideia greca, plasmando un'umanità razzialmente conscia delle sue origini e della

\footnotetext{
${ }^{8}$ CHAPOUTOT, Johann, Il nazismo e l'Antichità, Torino, Einaudi, 2017, p. 76 [ed. orig.: Le nazisme et l'antiquité, Paris, PUF, 2012].

${ }^{9}$ Ibidem, p. 81.
} 
gerarchia fra le razze. In una fortunata locuzione usata dallo stesso chapoutot il paradigma nazista, che ribalta quello classicista e romantico dell'ex oriente lux, può essere espresso con ex septentrione lux. E infatti alla declinazione in chiave nazista della filosofia greca, Platone in particolare, e della tradizione olimpica è dedicata un'ampia parte del volume. La resurrezione del canone antico non passava dunque solo attraverso la facile assimilazione alla grandezza politicomilitare di Roma, ma anche da quella intellettuale del platonismo, come antecedente storico dell'inegalitarismo hitleriano. La cultura greca letta dai nazisti diviene antinomica rispetto alla piccola morale del decalogo giudaico-cristiano e abbandona l'universalismo per realizzarsi nel proprio spazio politico: «i pedagogisti e i classicisti del Terzo Reich, fedeli in questo a Nietzsche, esprimono così la loro esecrazione per l'uomo scisso, incompleto, amputato, e concordano nella celebrazione dell'uomo totale, il voller Mensch greco» ${ }^{10}$.

Per sostenere questa tesi Chapoutot utilizza con abilità una vasta casistica dai risvolti inediti e grotteschi. È infatti così che in libri, opuscoli, sussidiari, si osserva la deformazione totale della storia, dell'epica e dell'archeologia: Penelope diviene una figura nordica, così come Sofocle; i templi greci sono l'evoluzione, attraverso perfezionamenti, della tipica casa germanica e ogni avanzamento culturale o tecnologico dell'antichità è frutto dell'intrinseco genio nordico presente nel sangue dei popoli antichi.

L'annessione simbolica e culturale non può non essere poi anche un'annessione territoriale. Così come gli antichi soldati-contadini del nord cercarono il loro Lebensraum a sud, allo stesso modo le armate hitleriane dovevano pretendere l'espansione a est. Si prenda ad esempio il caso dell'occupazione della Grecia nel 1941, che sotto quest'ottica nazista diviene soltanto l'ultima di una serie di ondate germaniche nella penisola mediterranea. I soldati tedeschi ritornano nella terra di Leonida, anche lui ariano, legittimando la conquista con precedenti storici e razziali. E al primo shock nello scoprire che la maggior parte dei greci è povera non ha occhi azzurri e capelli biondi (una «delusione di ordine economico ed estetico, ma anche razziale» ${ }^{11}$ ), fa seguito l'ovvia spiegazione "razziologica" (le invasioni hanno reso i greci moderni un meticciato inoperoso) e la conseguente ricerca del tipo razziale ariano, erede del greco antico.

Chapoutot passa in rassegna tutto: dai canoni estetici, all'architettura sorta fra il 1934 e il 1945, con una speciale attenzione ai manuali scolastici, al cinema e alle arti plastiche. Il suo lavoro ricostruisce a pieno come il lettore, il piccolo discente, lo studente accademico, lo spettatore e l'uomo politico nazista, siano posti davanti a un paradigma ideologico saldamente impiantato sulla rilettura hitleriana delle due grandi civiltà del mondo classico. L'asse su cui ruota il volume è quello di indagare come e in che misura i nazisti abbiano annesso sia gli spazi fisici della geografia che quelli culturali e ideali del passato, assegnando loro una funzione politica escatologica.

\footnotetext{
${ }^{10}$ Ibidem, p. 117.

${ }^{11}$ Ibidem, p. 97.
} 
Escatologia che si fonda più sulle rovine che sull'archeologia, sostiene Chapoutot, e che diventa soprattutto nella fase discendente della guerra un programma politico velato di lugubre messianismo: «Noi vinceremo. Se non vinceremo spariremo con onore fino all'ultimo uomo» ${ }^{12}$. Solo attraverso le rovine e la caduta Roma e Atene sono divenute immortali. Allo stesso modo, se il regime della croce uncinata, terza Roma, non avesse potuto trionfare sarebbe dovuto allora rovinare. In attesa della ventura ennesima parusia della razza nordica, nell'eterno ritorno, la sconfitta militare sarebbe stata mitigata dalla vittoria morale.

Il nazismo e l'Antichità è dunque un'ampia indagine sull'appropriazione del passato da parte dei nazisti, ricostruito con una vasta documentazione di oltre trecento libri, articoli, discorsi pubblici e pamphlet pubblicati durante il regime: dai testi di formazione politica alla letteratura pedagogica, dal diritto allo sport, dalla biologia alla filosofia, dall'antropologia alla storia e alla filologia, dalla linguistica alla geografia. Chapoutot lo corrobora inoltre con l'aggiunta di vari documenti d'archivio provenienti dal Bundesarchiv Berlin Lichterfelde dove è custodita la maggior parte del materiale ideologico del NSDAP e delle SS. Da questo libro emerge pertanto, in maniera elaborata e coerente, la falsificazione nazista dell'antichità e della storia, ed è al momento il lavoro più completo in materia. Le tre parti principali de Il nazismo e l'Antichità sono infatti coerenti nel dimostrare la pianificazione dietro quest'ideologia. L'antichità è dapprima annessa, successivamente ripetuta, contraffatta e imitata, infine riletta sotto la lente della nuova idea totalitaria.

Il nazismo attuò, e lo dimostra chiaramente Chapoutot, una rivoluzione culturale oltre che politica, per istituire un passato antico, immaginario ed esclusivamente tedesco, fondato sulla superiorità biologica della razza germanica, sorella di quella classica, da mettere in contrapposizione a quella semitica nella lotta secolare fra Oriente e Occidente, fra «principio nordico e principio semitico $»^{13}$ che aveva sopraffatto già Roma e Atene.

Il libro di Chapoutot ci restituisce, senza tralasciare nulla, il vero discrimine della logica totalitaria: l'assalto al mondo da parte dei nazionalsocialisti oltre che dalla mera geografia passava anche dalla conquista del passato.

\footnotetext{
${ }^{12}$ Ibidem, p. 387.

${ }^{13}$ Ibidem, p. 309.
} 


\section{L'AUTORE}

Alberto SCIGLIANO è dottorando in Storia presso l'Università di Teramo con una tesi sull'esemplarità politica d'Israele nella cultura ottocentesca. Si è interessato di sionismo socialista e identità israeliana. Attualmente si occupa di tradizione politica ebraica, repubblicanesimo in età moderna e uso delle fonti bibliche nel pensiero politico. È stato borsista presso l'Universitat Haifa dove ha approfondito lo studio della lingua e della cultura ebraica e svolto ricerche alla Nazarian Library.

URL: < http://www.studistorici.com/progett/autori/\#Scigliano > 